\title{
TTR
}

Traduction, terminologie, rédaction

\section{Relectures et traductions du Hamlet de Shakespeare par Yves Bonnefoy : de la ré-énonciation à la création}

\section{Stéphanie Roesler}

Volume 27, numéro 1, 1er semestre 2014

Lecture et traduction

Reading and Translation

URI : https://id.erudit.org/iderudit/1037118ar

DOI : https://doi.org/10.7202/1037118ar

Aller au sommaire du numéro

Éditeur(s)

Association canadienne de traductologie

ISSN

0835-8443 (imprimé)

1708-2188 (numérique)

Découvrir la revue

Citer cet article

Roesler, S. (2014). Relectures et traductions du Hamlet de Shakespeare par Yves Bonnefoy : de la ré-énonciation à la création. TTR, 27(1), 43-65.

https://doi.org/10.7202/1037118ar
Résumé de l'article

Toute traduction littéraire commence par la lecture, étape première de l'interprétation qui est déjà éminemment subjective. La traduction ne saurait prétendre à l'objectivité pas plus qu'à la transparence du traducteur, et ce plus encore dans le cas de la traduction de la poésie, où sens et forme sont inséparables. La voix du traducteur, expression même de sa subjectivité, sera nécessairement audible dans la traduction, qui est ré-énonciation. C'est ce que nous démontrerons ici à travers les cinq traductions du Hamlet de Shakespeare par Yves Bonnefoy, qui illustre comment, passant de la ré-énonciation à la création, il a su faire résonner sa propre voix de poète traducteur. Des notions théoriques empruntées à Barbara Folkart nous aideront à mettre en lumière le fait que la traduction de la poésie ne peut être que subjective, création d'un poème nouveau dans une autre langue, et finalement activité d'écriture. 


\title{
Relectures et traductions du Hamlet de Shakespeare par Yves Bonnefoy: de la ré-énonciation à la création
}

\author{
Stéphanie Roesler \\ Université Concordia
}

\begin{abstract}
Résumé
Toute traduction littéraire commence par la lecture, étape première de l'interprétation qui est déjà éminemment subjective. La traduction ne saurait prétendre à l'objectivité pas plus qu'à la transparence du traducteur, et ce plus encore dans le cas de la traduction de la poésie, où sens et forme sont inséparables. La voix du traducteur, expression même de sa subjectivité, sera nécessairement audible dans la traduction, qui est ré-énonciation. C'est ce que nous démontrerons ici à travers les cinq traductions du Hamlet de Shakespeare par Yves Bonnefoy, qui illustre comment, passant de la réénonciation à la création, il a su faire résonner sa propre voix de poète traducteur. Des notions théoriques empruntées à Barbara Folkart nous aideront à mettre en lumière le fait que la traduction de la poésie ne peut être que subjective, création d'un poème nouveau dans une autre langue, et finalement activité d'écriture.
\end{abstract}

Mots-clés: Yves Bonnefoy, Hamlet, Barbara Folkart, voix, traductionécriture

\begin{abstract}
All literary translation starts with the act of reading, which is the first, deeply subjective step of the interpretative process. Translation can neither be objective nor result in the translator's invisibility, even more so in the case of the translation of poetry, where form and meaning are inseparable. We need to hear the voice of the translator, as an expression of their subjectivity through their translation, which is a re-enunciation. This article illustrates how, through his five translations of Shakespeare's Hamlet, Yves Bonnefoy let his art of translation evolve from a reenunciation to a real creation, letting his own voice as a poet resonate through the translation and make itself heard. This analysis is based on the theoretical works of Barbara Folkart, who argues that poetic translation is profoundly subjective and aims to be the creation of a new poem in another language, that is to say a new act of writing.
\end{abstract}


Keywords: Yves Bonnefoy, Hamlet, Barbara Folkart, voice, translation as writing

\section{Introduction}

La lecture est l'étape première de la traduction, car avant de prétendre traduire un texte, il faut d'abord le comprendre, et comme l'a dit George Steiner, comprendre, c'est traduire. Dès cette étape, la subjectivité du traducteur est en jeu, car c'est avec tout ce qu'il est, avec son expérience, qu'il va lire le texte qu'il va traduire. Cependant, c'est lors de l'étape suivante, celle de l'élaboration du sens dans la langue d'arrivée, que le sujet traducteur se fait véritablement présent. Nous suivrons la théorie de Barbara Folkart (1991) dans Le conflit des énonciations pour appeler cette étape la ré-énonciation. Or, si l'on fait de la signification d'un texte à traduire le noyau pour ainsi dire «objectif» de ce texte, qu'arrivet-il dans le cas de la traduction de la poésie, qui est «forme du sens» (Meschonnic, 1999, p. 84), transmission d'un sens et d'une expérience à travers une forme, et inséparable de cette forme?

La lecture d'un texte poétique est un défi d'une autre nature pour le traducteur, qui ne peut se contenter de comprendre, mais doit éprouver le poème dans toutes ses dimensions signifiantes. C'est la traduction poétique qui nous intéressera ici, et plus particulièrement le cas de la traduction du Hamlet de Shakespeare par Yves Bonnefoy, ce dernier considérant Shakespeare avant tout comme un poète. Bonnefoy a traduit la plus célèbre des pièces de Shakespeare à cinq reprises en trente et un ans, si bien que chaque relecture du même texte a donné lieu à une traduction nouvelle. Or, à travers ces relectures et cette écoute, il a pu être sensible à différents aspects du texte et se l'approprier de manière toujours plus intime.

L'exemple de Bonnefoy traducteur de Hamlet nous permettra de montrer que le traducteur de poésie doit pratiquer un type bien particulier de lecture: une «lecture écrivante», pour reprendre les termes de Bonnefoy (2000, p. 10), qui va lui permettre de s'imprégner du poème original pour mieux s'en détacher, afin non pas de le reproduire, mais de créer un poème nouveau. De là, nous éclairerons sa poétique de la traduction par la pensée critique de Barbara Folkart dans son ouvrage Second Findings: A Poetics of Translation (2007). Folkart exige en effet du traducteur, et d'autant 
plus s'il est poète, qu'il produise une traduction "poétiquement viable $^{1} \gg$ (2007, p. 141), ce qui l'engage à écrire un poème nouveau.

\section{De la lecture à la ré-énonciation: lémergence de la voix du traducteur}

La ré-énonciation, telle que l'a définie Barbara Folkart dans $L e$ conflit des énonciations, est l'étape cruciale de la traduction, mais cette étape commence par la lecture. Or, qui dit ré-énonciation dit retour à l'origine... La traduction est ce processus qui vise à ré-énoncer la signification d'un texte écrit d'une langue dans une autre. Et lors de ce processus, si la langue est un filtre, la subjectivité du traducteur en est un second, car le traducteur met nécessairement du sien dans l'énoncé d'arrivée, qui apparaît dans «lépaisseur» de la traduction, comme l'explique Barbara Folkart, celle-ci trahissant «non seulement l'acte ré-énonciatif mais en même temps l'intervention du sujet ré-énonciateur » (1991, p. 384385). Ainsi, ce que le traducteur traduit est $s a$ lecture du texte. La "fidélité» est un leurre, car il y a nécessairement un certain degré d'appropriation du texte de départ, lorsqu'on parle de traduction littéraire. Le traducteur nous laisse entendre sa propre voix.

Mais arrêtons-nous justement sur ce terme de "voix», qui est précisément l'indice d'une subjectivité à l'œuvre dans le texte. Si la notion de voix est ambiguë et difficile à définir, elle se différencie de celle de style. La voix est en effet la manière dont une individualité manifeste son être-au-monde par le son, chaque voix étant unique, singulière. C'est en ce sens que l'on peut la définir comme ce «à quoi se reconnaît la manière d'un auteur» (Bernardet, 2007, p. 65). C'est la manière dont une subjectivité auctoriale se manifeste dans le verbe. La voix révèle que la pensée d'un individu et la manière dont elle prend forme dans les mots sont inséparables.

Dans le cas de l'écriture, la voix s'inscrit dans la langue à travers la syntaxe, la ponctuation, le rythme ou les cadences, les sonorités, les figures de style, les images, pour ne citer que quelques-uns des marqueurs qui rendent la voix d'un auteur identifiable et unique.

1. En anglais «poetically viable». Folkart explique à ce sujet: «[p]oetically viable translations-those that actually succeed in being poems-are the exception. Something that not even excellent translators can pull off all the time. Something that takes talent and a special combination of circumstances, I suspect, a strange mix of desire, perseverance, insight, flair, luck perhaps - and jouissance.» (2007, p. 141) 
Plus que des traits stylistiques, ces marqueurs révèlent l'être même de l'écrivain et son rapport au monde, dont la langue devient un substitut. Ce sont ces «traces laissées dans le produit par le faire producteur»(Folkart, 1991, p. 11) qui rendent perceptible la présence de l'auteur au sein même du texte. Or, la traduction est «travail de ré-énonciation/production» et "on ne saurait réénoncer sans $y$ mettre du sien» (ibid., p. 12; nous soulignons); dès lors, comme l'auteur, le traducteur fait lui aussi entendre sa voix, phénomène qui est le résultat même de l'acte de traduire. Cependant, «réintroduire le sujet traduisant dans le texte traduit, faire la part de la subjectivité» (et de la voix), c'est "paradoxalement le premier pas vers un plus grand respect du texte» (ibid., p. 14). «Sachant de quelles contingences est nécessairement entachée sa pratique, le traducteur sera moins tenté de tomber dans ce piège qui consiste à ériger en absolu sa propre subjectivité», à vouloir lire son propre être à travers le texte, précise Folkart (ibid.). L'on comprend ainsi que si la voix du traducteur est bien audible dans la traduction, celle-ci n'est pas superposable avec la voix de l'auteur, impossible à contrefaire. Cette dernière reste présente dans la traduction, mais de manière modifiée, pour ainsi dire, audible à travers le texte d'un autre, celui du traducteur.

\section{La voix, manifestation de la subjectivité du traduire}

Barbara Folkart a défini cette notion de voix comme une «isotopie subjective $^{2}$ », c'est-à-dire "l'ensemble des lieux non formalisés où l'énonciateur s'inscrit dans l'énoncé qu'il produit»; la notion de voix est ce qu'elle appelle une catégorie différentielle, qui relève $\mathrm{du}$ processus sémiologique de la verbalisation et qui est une «manière de concevoir et d'exprimer le monde et de communiquer sa conception du monde» (1991, p. 387). Cette voix se manifeste aussi bien dans la macro-forme discursive que dans la substance discursive $^{3}$, si bien que distinguer une voix, c'est «deviner

2. Folkart définit l'isotopie comme «ensemble de lieux textuels manifestant un trait (sémantique, lexical, phonémique, phonétique rythmique, etc.) dont la récurrence fait sens. L'isotopie peut être envisagée comme le paradigme dont la projection sur l'axe syntagmatique contribue à fonder la signifiance et la cohérence du texte» (1991, p. 445). Quant à l'«isotopie subjective (voix)», c'est «l'ensemble des lieux (subjectivèmes) s'indexant sur une seule subjectivité [...]» (ibid., p. 446).

3. De la substance, Folkart nous dit qu'elle est: «La matière mise en forme par une sémiotisation qui la récupère et la travaille. [...] Au niveau du 
l'existence d'un sous-idiome, ou système idiolectal, qui est le corrélat langagier de l'énonciateur» (ibid., p. 388). Chaque voix est unique et correspond à un idiome identifiable dans le texte d'un auteur ou d'un traducteur.

Dans Le conflit des énonciations, Barbara Folkart rejette la prétendue objectivité de cette opération ré-énonciative qu'est la traduction, et lie dès lors la notion de voix du traducteur, expression même de sa subjectivité, à celle de ré-énonciation : la voix est « une intervention du sujet ré-énonciateur», et elle permet de décrire "cette présence, dans le traduit du traducteur, "ré-énonciateur énoncé"» (ibid., p. 385). La notion de voix est, pour Folkart, l'une des idées clés de la traduction, au sens où le traducteur-réénonciateur ne peut être absent du texte traduit; au contraire, il s'y manifeste

sous forme de déviances, tant pragmatiques et référentielles que sémiologiques (les trois étant intriquées d'ailleurs), bref, sous forme d'indices de ré-énonciation [...]. Parmi toutes ces déviances, la voix du traducteur se dessine comme l'ensemble des lieux sémiologiques où celui-ci [le traducteur] ne réussit (ni ne saurait réussir) tout à fait à contrefaire la voix de l'auteur. (ibid., p. 394) ${ }^{4}$

Folkart contredit le mythe de la transparence du traducteur: sa propre voix, voix donc "seconde" puisquelle vient à la suite de la voix de l'auteur, se manifeste dans le texte de la traduction comme un ensemble hétéroclite de déviances, de sorte que Folkart peut conclure qu' «appliquée au ré-énonciateur, la notion de voix est essentiellement différentielle: la voix du traducteur se démarque de celle de l'auteur; la voix d'un traducteur donné se démarquera de

discours, la substance, c'est le syntagme tel qu'il se livre à l'observation (les structures déployées à la surface du texte), par opposition à sa sous-jacence enfouie, la macro-forme discursive. Manifestans, la substance actualise la forme sous-jacente (manifestatum) qui l'intègre au système ou au syntagme.» (1991, p. 453)

4. Notons les connotations péjoratives des termes «déviances", « contrefaire». Cependant, concernant le premier terme, celui de «déviances» (littéralement, «s'écarter de la voie»), Folkart précise dans une note: «Envisager la présence du traducteur comme un ensemble de déviances ou, ce qui revient au même, comme l'ensemble des lieux où s'inscrit dans l'énoncé la présence du ré-énonciateur", c'est se placer au point de vue de cette pratique de la traduction qui sera qualifiée de «mimétique» (p. 199 et sq.). 
celles des autres qui ont traduit le même corpus» (ibid., p. 395). Ainsi, tout en se superposant, la voix de l'auteur et celle du traducteur rivalisent entre elles.

Ces déviances dont parle Folkart, qu'elle appelle encore dissonances lorsque le traducteur tente de contrefaire la voix de l'auteur ${ }^{5}$, se font sentir à tous les niveaux du syntagme, aussi bien dans la forme que dans la matière discursives. C'est donc en s'insérant dans les «failles du syntagme » que le traducteur a «la possibilité de faire sentir sa voix, de marquer son interprétation» (ibid., p. 396), processus que Folkart semble évaluer de manière positive, car toute traduction/ré-énonciation est essentiellement différentielle, et se doit de l'être, si nous la suivons dans ses arguments. Or, ce phénomène qui consiste à marquer son interprétation nous semble particulièrement approprié pour caractériser la traduction de la poésie, car le traducteur fait nécessairement entendre sa propre voix dans le texte traduit, et c'est ce qui donne à la traduction «l'épaisseur» dont elle parle (ibid., p. 375). L'épaisseur de la traduction démentit les mythes «de la traduction transparente et du traducteur-absence» et atteste «que le traducteur y met du sien (et de son univers)» (ibid.). Nous défendrons, dès lors, l'idée que la présence du traducteur doit être sensible au cœur même du texte traduit, au sens où la traduction d'un poème n'est viable que si elle est création d'un poème nouveau, dans lequel la voix du traducteur est bel et bien audible.

\section{De la présence à la parole du traducteur}

Cette notion de voix nous permet d'ores et déjà d'établir un lien entre la théorie développée par Barbara Folkart et les idées

Lorsque la traduction, cessant de viser le mimétisme, se fait confiscation, (p. 413), re-création (p.417) ou création (p.419), «la voix du traducteur acquiert un statut comparable à celui de l'auteur». (p. 394, n. 108) Les mots de re-création ou de création nous laissent percevoir que le terme « déviances » n'est que partiellement négatif, quoiqu'il vise à souligner l'appropriation par le traducteur dont fait l'objet le texte de départ. Quant au terme «contrefaction », il renvoie à cette traduction dite "mimétique» et est donc plus nettement négatif (péjoratif) quant au processus qu'il décrit. En effet, Folkart cherche avant tout, dans son ouvrage, à polémiquer avec la notion de «fidélité » (p. 11). Dans tous les cas, notons que le ton de Folkart diffère de celui de Bonnefoy dans sa description de la traduction et de la retraduction, ce dernier étant bien plus nettement positif.

5. Processus qu'elle condamne, d'où la connotation négative du terme «dissonance». 
d'Yves Bonnefoy, malgré la différence de leurs approches et de leurs tons. Pour Bonnefoy, la poésie est "parole», ce qui la situe sur le même plan de l'oralité que la voix, et c'est un indice de l'importance que Bonnefoy donne au son et au rythme dans la poésie. De plus, traduire un auteur consiste essentiellement à traduire une voix, et plus encore lorsqu'il s'agit de traduire un poète, car «un poème, c'est une voix» (Bonnefoy, 1993b, p. 66); cette voix a une fonction médiatrice et instaure un dialogue avec autrui. Selon Bonnefoy, le traducteur qui souhaiterait rendre la voix d'un poète devra donc être capable de percevoir et de reconstruire le caractère indissociable d'une pensée et de la forme qu'elle prend, caractère qui est le cœur même de la poésie.

Dans un premier temps, Bonnefoy applique le principe bermanien d'accueil de l'Étranger, car il a bien conscience que le respect du texte original passe par une écoute attentive de la parole de l'Autre - ou, dirons-nous, de cet instrument de musique (pour reprendre une métaphore de Bonnefoy) qu'est sa voix. Cependant, traduire, c'est aussi s'essayer à faire résonner cette voix à l'aide d'un instrument différent, d'abord en ce que le langage musical (si l'on file la métaphore) auquel il a recours n'est pas le même; il s'agira également pour le traducteur, au cours de cette réénonciation qu'est la traduction, de répondre au poète qu'il traduit et de laisser entendre sa propre voix. Car finalement, traduire consiste à articuler une réponse au poème original, réponse qui incarne l'écoute personnelle que le traducteur a pu faire de cet original. Mais dès lors, que se passe-t-il dans le cas de multiples ré-énonciations, donc de relectures successives? C'est ce que nous tenterons d'examiner dans le cadre des cinq traductions de Hamlet par Yves Bonnefoy.

\section{De la voix de l'auteur à la voix du traducteur: le Hamlet de Shakespeare traduit par Yves Bonnefoy}

Nous avons analysé, dans le cadre de notre thèse de doctorat, les variantes stylistiques des cinq traductions du Hamlet de Shakespeare par Yves Bonnefoy, publiées respectivement en 1957, 1962, 1965, 1978 , et 1988. D'une version à l'autre, les changements sont plus ou moins importants, mais chaque traduction est différente de la précédente, donc unique. À l'issue de notre étude détaillée des cinq traductions de Bonnefoy, la difficulté d'établir le sens de leur évolution est cependant apparue : celles-ci n'évoluent pas de façon 
unilatérale et constante. Ce que ces traductions reflètent, ce sont davantage les tâtonnements de Bonnefoy, car tantôt il se démarque de la traduction précédente et innove, tantôt fait marche arrière et revient à une traduction antérieure, oscillant entre le respect de la voix de Shakespeare et l'affirmation de sa propre voix.

\subsection{Le lexique}

Par différents aspects cependant, la traduction évolue de manière double ou paradoxale, ce qui reflète cette oscillation. Ainsi, dans le domaine lexical, si à certaines occasions Bonnefoy s'efforce de trouver le meilleur équivalent sémantique au terme anglais, son respect des termes du texte n'est bien souvent que partiel; parfois, il s'écarte nettement du texte original, comme s'il cherchait davantage à évoquer celui-ci plutôt qu'à le rendre par un strict littéralisme. Souvent, l'écart avec le texte original est prétexte à l'inventivité langagière et poétique. Parfois, Bonnefoy semble se concentrer sur la matérialité sonore des mots et se révèle virtuose dans l'art des assonances et des allitérations. Ainsi, pour prendre un exemple, au vers 116 de l'acte $\mathrm{I}^{6}$, scène 1 , le verbe "gibber» est d'abord traduit par "chuchoter», puis il préfère "balbutier» en 1978, mot qui, par une allitération en -b, respecte l'effet sonore produit par «gibber».

Enfin, Bonnefoy a recours à différents procédés de traduction pour rendre le texte shakespearien, explorant les ressources propres au français et affirmant le sens qu'il souhaite donner au texte. Prenons l'exemple du vers 73 de l'acte I, scène 2 : l'expression "Passing through nature to eternity», ou plus précisément la locution verbale "passing through", exprime l'idée d'un passage, d'une traversée, mais aussi d'un départ; or, Bonnefoy a jugé plus importante l'idée de départ, d'un mouvement de progression de la nature humaine, et a choisi d'étoffer "passing through" par les verbes «quitter» et «regagner» pour écrire «quitter notre condition pour regagner l'éternel». On a donc deux propositions au lieu d'une, et la finale vient concrétiser l'idée de progression.

6. La numérotation des vers est celle de la traduction de Jean-Michel Déprats (Shakespeare. Hamlet. Préface, dossier et notes de Gisèle Venet, trad. de Jean-Michel Déprats, établissement du texte anglais H. Suhamy, Gallimard, «Folio Théâtre», 2004, 405 p.), qui a également pour source le second quarto de Hamlet, les traductions de Bonnefoy ne comportant pas de numérotation. 
Cette traduction fort peu littérale respecte néanmoins la substance signifiante de l'expression originale, mais frappe surtout par sa fluidité et son équilibre, qui témoignent d'un réel travail sur la langue d'arrivée.

Dans d'autres cas, Bonnefoy est nettement infidèle au lexique du texte et rend palpable, par les mots qu'il choisit en français, son interprétation du texte. Pour ce faire, il use parfois de la clarification. Ainsi, le vers 122 de l'acte $V$, scène 2 , «Is't not possible to understand in any other tongue», est d'abord traduit par «Ne pouvez-vous pas comprendre chez un autre que vous votre façon de parler?» (1957 à 1959), ce qui permet à Bonnefoy de clarifier ce que signifie «other tongue» dans le contexte: une autre langue, au sens d'une autre façon de parler. La seconde version (1962 à 1988) reste sensiblement la même: «Ne comprenez-vous pas votre parler chez un autre?», mais il nous faut ici remarquer que, outre la clarification sémantique qu'elle opère, la traduction de Bonnefoy met moins l'accent sur un problème de langue que sur l'impossibilité de la communication entre Hamlet et le courtisan, entre deux mondes, entre le soi et l'autre... Ce qui renforce sa solitude, une solitude qui s'exprime au cœur de la langue, et qui rapproche d'ailleurs Hamlet du traducteur?

\subsection{La création d'un Hamlet français}

Bonnefoy semble, en sondant les ressources de la langue française, vouloir produire un texte qui ne soit pas une pâle imitation de l'original, mais soit une œuvre à part entière. Par ailleurs, il se rend au caractère racinien et au désir de clarté de la langue française, comme lorsqu'il supprime les doublets shakespeariens et qu'il va dans le sens d'un allègement quantitatif afin de resserrer le tissu lexical du texte. Ainsi, à l'acte I, scène 1 , vers 68 , le doublet «But in the gross and scope 8 of mine opinion" est simplifié en "Mais ma première idée» (1957 à 1988). À l'acte II, scène 2, vers 55, "The head and source of all your son's distemper» est traduit par

7. Or, comme nous l'avons montré dans notre thèse, Bonnefoy semble s'identifier au personnage d'Hamlet, ou plus précisément à Shakespeare et à son questionnement existentiel à travers Hamlet, qui est pour lui le personnage dans lequel Shakespeare s'est incarné le plus nettement tout en se découvrant à travers celui-ci. En outre Hamlet incarne le poète, celui qui ne croit plus au sens, ni au langage comme véhicule de ce sens. (Roesler, 2010).

8. Nous soulignons les termes en italiques dans les extraits de Hamlet et les traductions de Bonnefoy correspondantes. 
«Lorigine des maux de votre fils» jusqu'en 1978, puis par «la cause du dérangement de votre fils» (1988); dans les deux cas donc, le doublet «head and source» n'est pas préservé. ${ }^{9}$ Bonnefoy travaille avec les caractéristiques de sa langue, ne cherchant pas à reproduire Shakespeare, mais à le recréer en français. Au foisonnement lexical de Shakespeare, il oppose la brièveté d'expression et un texte toujours plus dense. Remarquons en outre qu'au fil de ses cinq traductions différentes, le traitement que Bonnefoy réserve aux doublets a évolué: si dans un certain nombre de cas il les avait d'abord conservés, il décide ensuite de les supprimer. En cela, il assume plus nettement son choix de la condensation, donc d'une traduction plus créative, et façonne le texte en fonction de sa poétique propre.

\subsection{L'empreinte de la poésie de Bonnefoy: simplification, densification}

On peut aussi reconnaître, dans la manière dont Bonnefoy retravaille le texte selon le principe d'une plus grande simplicité, l'une des tendances fondamentales de sa propre poésie. La poésie de Bonnefoy évolue en effet dans le sens de la brièveté, de la condensation pour, par un nombre restreint de vocables, exprimer le sens. En effet, les notions de «simple» et de «sens» se complètent, sont interdépendantes, comme l'explique Michèle Finck (1989). Si la poésie de Bonnefoy est une quête du sens, cette quête passe par la simplicité. Le simple est «l'exigence d'une parole qui cherche à prendre terre dans la vie ordinaire ", écrit Finck (ibid., p. 6), qui conçoit précisément la poésie de Bonnefoy «comme un approfondissement progressif du mot "simple"» (ibid., p. 9). Ainsi, par sa quête d'un langage toujours plus simple dans ses traductions, il montre qu'il traduit en poète et que son activité de traducteur n'est pas séparable de son activité de poète. Il cherche, en fait, à rester en accord avec sa conception de la parole poétique, qui est vouée à l'échange avec autrui. Ainsi, même lorsqu'il semble servir le texte de Shakespeare et dialoguer avec celui-ci, il suit ses propres tendances poétiques.

9. Thélot fait remarquer que ce resserrement du lexique dans l'œuvre de Bonnefoy «a une fonction spirituelle» et correspond à une «indispensable simplification du réel» (1983,p. 149). Il cite à l'appui de ses propos Bonnefoy se réclamant de Racine et de «l'idée racinienne de la parole» qui consiste à «simplifier la conscience, de nous attacher à quelques pensées qui sont bien sûr les plus graves» (L'Improbable, p. 160. Cité par Thélot, 1983, p. 149). 
La remarque qu'Antoine Berman faisait à propos des traductions de John Donne par Bonnefoy nous semble valable aussi dans le cas de la traduction de Hamlet. Reprenons ses propos:

Dire que Bonnefoy laisse ce côté oratoire [de la phrase de Donne], c'est dire qu'il simplifie et densifie légèrement le vers de Donne. Ce en quoi, il va aussi, nous le savons, dans le sens du poète, qui aime le "compact», le «dense», le «simple». Mais cette décision de simplification et de légère densification est une décision de traducteur [...]. C'est un traduire qui se laisse cerner très nettement, et d'abord par les quelques caractéristiques que j'ai évoquées, et qui ne sont pas propres à Bonnefoy seulement. Ce traduire produit ce que Goethe appelait la Verjüngung (rajeunissement) ou la Verfrischung (régénération, rafraîchissement). Les deux poèmes de Donne nous apparaissent comme neufs, comme jeunes [...]. Cette impression de rajeunissement des poèmes est due à l'abandon de toute poétisation, de toute rhétorisation [...] et aux légères procédures de simplification et de densification observables. La Verjüngung est un mouvement temporel: la traduction de Bonnefoy présentifie Donne, le met dans un «now» qui est à la fois le sien (temporellement et originairement) et le nôtre, et le sort du passé antiquaire dans lequel le temps lui-même et les traductions passéistes l'avaient mis. (Berman, 1995, p. 196)

Selon Berman, la simplification et la densification opérées par Bonnefoy sur le poème de Donne permettent au poème de revivre: elles réalisent un rajeunissement qui se fait «reviviscence», selon le terme employé par Berman (ibid.,p. 197). Par cette reviviscence, le poème de Donne a acquis «la liberté, la densité, la diaphanéité, la jeunesse et la légère prosaïcité du poème moderne» (ibid.), prosaïcité que l'on retrouve dans la poésie de Bonnefoy, dans son ton, sa concrétion, ses mots quintessenciés.

Or, il nous semble que Bonnefoy simplifie et densifie également le vers de Shakespeare, ce qui témoigne du fait qu'il traduit en poète, mais aussi d'un choix traductif. La légère prosaïsation dont parle Berman est aussi sensible dans les traductions de Hamlet, écrites dans une langue évoluant vers la simplicité d'expression et de diction, écartant les artifices rhétoriques ou stylistiques pour se faire plus dense. Cette 
densité est aussi palpable dans le fait que Bonnefoy élague le texte shakespearien, si bien que les phrases françaises sont soit de même longueur que les phrases anglaises, soit plus courtes. Il pratique ainsi «l'appauvrissement quantitatif», l'une des treize tendances déformantes de la traduction répertoriées par Berman, qui consiste précisément à «attenter au tissu lexical de l'œuvre, à son mode de lexicalité, le foisonnement» (1999, p. 59). Bonnefoy nous offre une œuvre nouvelle, écrite dans le bic et nunc de la langue française, avec les moyens de sa poétique propre, et qui rajeunit tout à la fois le poème de Shakespeare.

\subsection{Modification de la nature et de la forme des mots}

La «fidélité paradoxale» dont Bonnefoy fait preuve se manifeste aussi dans ses fréquentes modifications de la nature et de la forme des mots. Bonnefoy rend la substance signifiante de l'original, mais en ne respectant pas strictement la lettre du texte, puisqu'il change de catégorie grammaticale et modifie le nombre de mots. Ainsi fait-il une grande place aux noms, qui sont la catégorie grammaticale qu'il privilégie dans sa propre écriture ${ }^{10}$, comme l'explique Jérôme Thélot: «Puisqu'elle vise l'intériorisation des substances, l'œuvre de Bonnefoy accorde au substantif la prédominance sur les verbes et les adjectifs » (1983, p. 130). De nombreux adjectifs shakespeariens se voient donc transposés en noms en français ${ }^{11}$. La traduction atteste aussi d'un emploi notoire du singulier (surtout des noms singuliers) là où l'original emploie un pluriel. Là encore, il faut rappeler que les grands noms singuliers sont extrêmement présents dans

10. En cela, il reprend une ces conventions linguistiques du français telles qu'analysées par Vinay et Darbelnet, à savoir, «la prédominance du substantif en français» (1977, p. 102). Louvrage de Vinay et Darbelnet, quoique daté et souvent contesté, continue dêtre un point de référence pour certains traductologues concernant précisément certaines conventions linguistiques de la langue française qui continuent d'être repérées par l'analyse comparée du français avec l'anglais.

11. Ladjectif «thorny» (acte I, scène 3, vers 48) est traduit d'abord par «épineux», puis par le substantif « ronces » (dès 1978). À l'acte I, scène 4, vers 61, «a more removed ground» est traduit d'abord par «un lieu plus écarté», puis «un lieu plus à l'écart» (à partir de 1978); le participe passé employé 
la poésie de Bonnefoy ${ }^{12}$. Or, dans la mesure où la traduction évolue vers un emploi toujours plus net des substantifs et que se maintient la préférence pour le singulier, on peut dire que Bonnefoy poète rend sa voix de plus en plus audible dans celle-ci tout en réaffirmant les tendances propres à sa langue. Il illustre les propos de Folkart lorsque celle-ci explique que «la voix du traducteur se dessine comme l'ensemble des lieux sémiologiques où celui-ci [le traducteur] ne réussit (ni ne saurait réussir) tout à fait à contrefaire la voix de l'auteur» (1991, p. 395). Cependant, l'inscription de la voix de Bonnefoy dans la traduction relève autant d'une impossibilité de contrefaire la voix de Shakespeare que d'un choix véritable. Davantage, c'est l'affirmation de ce choix que l'évolution des traductions rend palpable.

\subsection{Variations syntaxiques}

Dans le domaine de la syntaxe, l'évolution de la traduction apparaît d'abord double, mais, ultimement, Bonnefoy a tendance à s'écarter du texte original. D'un côté, Bonnefoy rend sensibles ses efforts pour donner un équivalent aux nombreuses irrégularités syntaxiques propres au texte shakespearien ainsi qu'à la mise en valeur de certains mots qui en découle. Toutefois, plus nombreuses sont les occurrences où Bonnefoy modifie la syntaxe, les unités sémantiques, l'ordre des propositions ou encore ajoute de la ponctuation. Là aussi, il nous semble qu'il opte pour une poésie légèrement prosaïque, dans le sens où il choisit l'abandon de la complexité syntaxique pour aller vers un texte plus fluide. Il travaille à sa guise la syntaxe française pour donner à la traduction sa propre texture.

comme adjectif «removed» est donc remplacé par un nom. Plus loin, à l'acte III, scène 2 , vers 76 , l'expression «damned ghost» est rendue par «spectre de l'enfer»: «damned» devient donc le nom «enfer», etc.

12. Les exemples suivants en témoignent: aux vers 102-103 de l'acte II, scène 1, Shakespeare écrit: «As oft as any passions under heaven/That does afflict our natures», ce que Bonnefoy rend par «A chaque fois qu'ici-bas nos passions/Affligent notre nature», où l'on remarque le singulier «notre nature» (1988). À la scène 2 du même acte II,l'expression du vers 493, «The very faculties of eyes and ears», est traduite par «Notre oreille et notre œil», puis «De l'œil et de l'oreille». Dans les deux cas donc, deux noms pluriels sont rendus par des singuliers. 


\subsection{Le travail poétique des traductions}

Dans le domaine de la poétique, les traductions de Bonnefoy semblent là encore se caractériser par deux tendances opposées: d'un côté, son travail poétique va dans le sens d'un rendu du texte shakespearien, de l'autre, les interventions de notre traducteur sont nombreuses. Ainsi, les caractéristiques sonores et musicales du texte sont assez minutieusement respectées par Bonnefoy: les allitérations et les assonances sont toujours mieux rendues, soit de façon stricte ${ }^{13}$, soit par la recherche de sons équivalents. Pour illustrer ce dernier phénomène, on peut mentionner l'acte III, scène 1, vers 75: la traduction française «De rien qu'un coup de dague » comporte une allitération en - $\mathrm{d}$ qui vient répondre à l'allitération en -b de l'original: "With a bare bodkin». Quant à la recherche d'équivalence en matière d'assonances, elle peut être illustrée par l'exemple de l'acte IV, scène 7, vers 163-164: "There is a willow askant the brook/That shows his hoary leaves in the glassy stream»: alors que ces vers comportent une assonance en -o, Bonnefoy crée, lui, une assonance en -i, et ce dans toutes les versions. Cela donne en 1988: "Tout auprès d'un ruisseau un saule se penche/Qui mire dans les eaux son feuillage gris». Ce travail sur les assonances et les allitérations n'est pas vraiment surprenant, à dire vrai, car Bonnefoy accorde une grande importance à la dimension sonore de la poésie, ou plutôt de la "parole» poétique pour reprendre son expression (1993a, p. 210 et 212, par ex.). Ainsi, son travail sur les sonorités se fait-il de plus en plus sensible au fil des traductions.

Si Bonnefoy respecte toujours davantage les parallélismes, les répétitions et les jeux lexicaux, il ajoute des parallélismes ${ }^{14}$ et des jeux avec le lexique, comme s'il voulait rendre la voix de Shakespeare toujours plus audible. Cependant, les répétitions sont aussi un trait caractéristique de sa propre poésie, car «pour

13. Ainsi, à l'acte I, scène 1 , aux vers $68-69$, les sonorités en -p et -s des vers de l'anglais: "But in the gross and scope of mine opinion/This bodes some strange eruption to our state» sont particulièrement bien rendues dans la version de 1978-88.

14. L'acte IV offre plusieurs exemples d'ajouts de parallélismes en français. À la scène 4, par exemple, le vers 51 «What is mortal and unsure» est traduit de manière plus verbeuse et surtout avec la création d'un parallélisme non présent dans l'anglais (en 1978-88), ce qui donne «Son être même, pourtant précaire, pourtant mortel». 
Bonnefoy, la répétition "simplifie" la langue, qu'elle concentre autour d'un noyau. Elle est vécue par le poète comme un redoublement de sens» (Finck, 1989, p. 354). Michèle Finck la rattache donc à la quête du simple et du sens sous laquelle elle place l'œuvre de Bonnefoy. Celui-ci conserve donc un trait de l'original qui est aussi caractéristique de sa propre écriture poétique.

Enfin, Bonnefoy s'efforce vraisemblablement de rendre le rythme du texte, qui est celui du pentamètre iambique, et les accents de celui-ci. Il met à profit la syntaxe, la ponctuation, les choix lexicaux et stylistiques ${ }^{15}$ pour ce faire, de sorte que l'on peut dire là aussi que la traduction évolue vers une plus grande proximité au texte shakespearien. Il travaille l'hendécasyllabe, un vers de onze pieds, pour lui donner la même souplesse et le même élan que ceux du pentamètre iambique. Cependant, ce choix du mètre impair comme équivalent du pentamètre est un choix qui lui est propre... et il en est venu à utiliser l'hendécasyllabe dans sa propre poésie. Traduire Shakespeare l'a aidé à trouver sa propre voix de poète, et en retour, cette voix se fait plus nettement audible dans ses traductions.

\section{La traduction de la poésie comme écriture d'un poème nouveau}

Les fruits de notre analyse peuvent cependant être interprétés autrement. Plutôt que d'affirmer que les traductions de Hamlet par Yves Bonnefoy évoluent de manière paradoxale ou double, il serait plus juste de dire que ce qui devient de plus en plus sensible, c'est le fait que, pour Bonnefoy, être véritablement respectueux du poème original consiste à lui donner un équivalent dans la langue d'arrivée, à écrire un nouveau poème en français ayant la même

15. À l'acte I, scène 5 , vers 186 , l'anglais dit: «And still your fingers on your lips I pray», les accents tombent sur «fingers» et «lips», ce que Bonnefoy rend en intervertissant l'ordre des propositions et traduisant en 1988 par « Et vous, je vous en prie, bouche cousue », mettant en valeur l'expression «bouche cousue». Autre exemple: à l'acte III, scène 3 , le vers 94 , «Ah that his soul may be as damned and black», comporte des accents sur "damned et black», deux adjectifs également mis en valeur par la sorte de «découpage» produit par les consonnes - $d$ et -b. Bonnefoy rend cela en intervertissant l'ordre des adjectifs, mais aussi en insérant l'adverbe "alors", qui marque une pause entre ces deux adjectifs ainsi soulignés: «Et que son âme soit aussi noire, alors, et maudite» (1978-88). 
qualité poétique que l'original. C'est en ce sens que la notion de ré-énonciation nous est utile, car Bonnefoy est loin de faire fi de sa subjectivité de traducteur, bien au contraire, et il rend audible sa voix de traducteur.

Par ailleurs, s'il est des domaines dans lesquels Bonnefoy semble tiraillé entre deux tendances - l'écoute de la voix de Shakespeare et l'affirmation de sa propre voix -, il est des aspects de ses traductions qui restent constants tout au long de celles-ci et qui révèlent nettement son parti-pris et l'affirmation de sa propre conception de la langue et de sa poétique. D'une part, comme nous l'avons expliqué plus haut, rappelons qu'au foisonnement lexical de Shakespeare, il oppose la brièveté d'expression et un texte toujours plus dense, et qu'il n'hésite pas à changer la forme grammaticale des mots, en donnant la préférence au substantif.

Bonnefoy délaisse parfois davantage le texte shakespearien pour laisser place à sa propre créativité; évoquons, par exemple, dans le domaine lexical, le fait que Bonnefoy gomme les références vulgaires et la « trivialité» rencontrées dans le texte de Shakespeare, s'affirmant en cela comme un disciple de Racine et un héritier du classicisme français. Il a d'ailleurs reconnu plus tard la remise en question de sa propre écriture que cette confrontation à la trivialité shakespearienne a entraînée:

Traduisant Shakespeare, j'ai eu pour ma part à affronter la question de l'acceptation par l'auteur d'Hamlet d'images on ne peut plus triviales au sein même de la parole tragique, ce qui révèle un rapport de la conceptualisation au monde tout autre que dans le théâtre de Racine, tout autre que chez Victor Hugo ou Claudel: et ce fut donc devoir repenser beaucoup de choses depuis ma lecture du classicisme, obligé d'avouer ses torts, jusqu'à des aspects de ma propre vie. (Bonnefoy, 2004, p. 75)

Néanmoins, jusqu'en 1988, si cette confrontation a eu lieu, elle ne s'est pas concrétisée par un respect de cette dimension triviale du texte, qui disparaît largement dans la traduction. On peut rapprocher ce non-respect de la trivialité du texte shakespearien de la tendance déformante de l'ennoblissement, telle que définie par Berman, «rhétorisation embellissante» visant à rendre la traduction plus belle que l'original (1999, p. 58). 
Dans le domaine poétique, Bonnefoy n'est pas littéralement fidèle aux métaphores shakespeariennes et crée volontiers des métaphores nouvelles; les traductions évoluent dans le sens de cette créativité et la version de 1988 se distingue en la matière. Par ailleurs, la présence poétique de Bonnefoy dans la traduction est aussi rendue sensible par l'apparition de vocables de sa poésie, ces grands substantifs qu'il utilise de façon récurrente, et par l'association de mots concrets et abstraits qui est caractéristique de sa propre écriture. Enfin, le travail que Bonnefoy a effectué sur le rythme dans ses traductions du texte de Shakespeare est révélateur, car le rythme est sans doute à ses yeux l'un des enjeux les plus fondamentaux de l'écriture poétique.

\section{Le rythme comme inscription d'une subjectivité dans le langage}

La référence à Henri Meschonnic (1999) s'impose ici: pour Meschonnic, le rythme est le révélateur même de l'inscription du sujet dans son discours, l'indice d'une poétique. Meschonnic définit le rythme dans le langage «comme l'organisation du mouvement par la parole, l'organisation d'un discours par son sujet et d'un sujet par son discours. Non plus du son, non plus une forme mais du sujet» (ibid., p. 115). Le rythme atteste de la recréation d'un continu entre le sujet et son discours, de l'inscription sensible d'un sujet dans son texte, car il vient du corps, donc du plus profond de l'être; par le rythme, la forme fait sens. Selon Meschonnic, «le mode de signifier, beaucoup plus que le sens des mots, est dans le rythme, comme le langage est dans le corps [...]. C'est pourquoi traduire passe par une écoute du continu. Subjectivation pour subjectivation» (ibid.).

En s'inscrivant par le rythme dans son texte, le traducteur s'y révèle présent comme sujet. Il recrée une poétique, transformant ainsi «le mode de signifier» (ibid., p. 102); il réalise ce que Meschonnic appelle une «traduction-texte» (1973, p. 313), c'està-dire une traduction qui soit elle-même un texte, non pas une vaine imitation de l'original, mais la recréation de ce continu sans lequel elle ne serait que forme vide et désincarnée. Créer un texte nouveau en une époque et un autre lieu, voilà l'enjeu essentiel du traduire, mais qui n'est possible que si le traducteur se révèle présent dans son texte; et plus le traducteur s'inscrit comme sujet dans la traduction, plus il parvient à recréer un texte nouveau à une 
autre époque et dans une autre langue. «Poétique pour poétique» (Meschonnic, 1999, p. 27).

En s'investissant ainsi dans le travail rythmique de sa traduction, Bonnefoy révèle donc sa conception de la traduction comme écriture d'un texte nouveau, recréation d'un continu, invention d'une poétique en réponse à la poétique de l'original. Pour lui comme pour Meschonnic, l'éthique du traduire passe par la poétique. Respecter le texte revient à faire texte: écouter Shakespeare, c'est savoir lui répondre.

\section{La création d'un poème nouveau dans la langue d'arrivée}

Bonnefoy a accompli ce «faire-œuvre-en-correspondance» considéré par Berman comme la tâche la plus haute de la traduction (1995, p. 94). Davantage, pour être éthique, la traduction de la poésie doit réaliser la poéticité, au sens où la traduction d'un poème ne peut être que la création d'un poème nouveau, fruit de la lecture du traducteur, de son interprétation, et de son maniement de sa propre langue. Les propos de Bonnefoy confirment cette interprétation: «[1]a traduction de la poésie a pouvoir d'être un dialogue, ou même une collaboration. Elle recommence l'œuvre en recommençant elle-même. Elle fait du poème une conséquence autant qu'une cause» (2003, p. 205). Collaboration et recommencement, la traduction de la poésie consiste à créer un poème nouveau, né d'un poème premier, tout en ouvrant une nouvelle voie en poésie.

Or, Bonnefoy a évolué, dans sa pratique de traducteur tout autant que dans ses commentaires sur celle-ci, vers une affirmation plus nette d'une poétique de traducteur qui consiste à créer une œuvre nouvelle dans la langue d'arrivée, surtout dans le cas particulier de la poésie. C'est en ce sens qu'il est pertinent de rapprocher la poétique de traducteur d'Yves Bonnefoy de celle de Barbara Folkart, dans son livre Second Findings: A Poetics of Translation. Ce livre est d'une tonalité tout autre que Le conflit des énonciations; tandis que dans le premier Folkart exposait plutôt une théorie de la traduction, dans le second, elle se détache de la sémiotique et de la linguistique pour puiser davantage dans son expérience de lectrice de traductions et de traductrice. Elle engage le traducteur à produire un poème nouveau, qui ait sa propre valeur poétique, ce qu'elle appelle «a poetically viable translation»: 
Only by re-enacting - or better, re-inventing - the "ratio difficilis ${ }^{16}$ " invented by the source-language poet can the translator - the target-language writer - produce a text that not only resonates with the original but is capable of functioning as a free-standing poem. (Folkart, 2007, p. 11)

Pour Folkart, le traducteur est bien plus qu'un simple lecteur, il est ce faber qui doit s'engager dans la fabrique d'un texte poétique, c'est-à-dire qu'il ne s'agit pas pour lui de répéter ce qui est dit par l'original, mais d'écrire $e^{17}$ un poème nouveau ${ }^{18}$. Écrire un poème nouveau dans la langue d'arrivée est bien différent de reproduire l'original de façon mimétique. De la même manière, Bonnefoy différencie deux types de traducteurs: les traducteurs que l'on pourrait qualifier de "passifs» et les autres qui «vont moins lire un poème que, le prenant dans l'espace de leur parole, commencer d'écrire avec lui, en lui, accompagnant son auteur mais aussi l'attirant sur leurs chemins» (2000, p. 9-10). Ainsi la traduction telle que Bonnefoy la conçoit doit-elle être cette «lecture écrivante», ce qui signifie que le traducteur doit non seulement lire, mais commencer à traduire à l'aune de sa propre expérience et de sa propre poétique. Comme Folkart, Bonnefoy nous invite donc à revoir l'idée que nous nous faisons de la traduction de la poésie, car si l'on parle d'une traduction «altruiste », "qui s'efface devant le texte parce qu'il ne s'agit que d'en offrir au lecteur autant d'aspects que possible» et d'une traduction «intéressée», «du fait que son auteur reste dans le champ de son écriture déjà en cours», il suggère que ce ne sont pas deux pôles opposés mais deux activités qui communiquent (ibid., p. 14-15). Cessons de séparer le pôle éthique du pôle poétique, pour employer des termes bermaniens, nous dit Bonnefoy, car la traduction d'un poème

16. "The "ratio difficilis" is a synergistic phenomenon, deeply rooted in the textures of its raw material: sound, prosody, imagery, denotations, connotations, intertextualities converge against all odds and enter into resonance, forming a nexus which, more often than not, cannot be replicated through the raw material of another language.» (Folkart, 2007, p. xi)

17. Nous soulignons.

18. Citons les propos de Folkart: «I would like now to demonstrate an alternative approach to the practice and theorisation of translation: a proactive approach that emphasizes writing, rather than reading: l'à-dire (sic) rather than the déjà-dit; the poem-to-come rather than the one that's already there. The name of the game, as far as I am concerned, is to write a target-language poem, as opposed to repeating the source-language poem.» (2007, p. 17) 
est nécessairement une activité d'écriture, si bien qu'il affirme: «Toute traduction d'une œuvre de poésie véritable peut bien se vouloir aussi scientifique que possible : si elle n'est pas simplement médiocre, elle sera naturellement un acte de poésie» (ibid., p. 15). Ainsi, Bonnefoy décrit-il le traducteur-écrivain:

[II] sait que la poésie est un acte, il sait que c'est cet acte qu'il faut traduire. Et il a donc compris que ce ne sera possible qu'au plan où lui-même, cessant d'être un récepteur passif, et voué à la seule intellection, va prendre en mains sa propre existence, dans une écriture à son tour, où celle du poète qu'il veut traduire jouera le rôle de visiteur écouté, de guide. Le traducteur de la poésie se doit d'être un poète, obligé à soi autant qu'à l'auteur. C'est précisément pour cela et comme cela qu'il n'est pas le lecteur ordinaire, celui qui ne songe pas à traduire. Il est autre. Est-il davantage? Disons qu'il se donne une tâche qui va le requérir davantage. (Bonnefoy, 2004, p. 74)

Et cette tâche a de particulier qu'elle se veut écriture, recherche de poésie. Bonnefoy a fait ce choix d'envisager la tâche de la traduction poétique comme «une forme de la création poétique et une réflexion sur le devenir de la poésie» (2004, p. 79).

En somme, pour Bonnefoy comme pour Folkart, écrire de la poésie et traduire de la poésie ne sont pas deux activités séparées: elles relèvent toutes deux d'un acte de création. Dès lors, la traduction de la poésie comporte nécessairement une dimension «domesticatrice» (Venuti, 1995) et appropriatrice, d'une part car le traducteur doit "adapter», pour ainsi dire, le poème à sa propre langue-culture, et d'autre part car c'est nécessairement à l'aune de sa propre poétique qu'il traduit. Ainsi Folkart envisage-t-elle la traduction de la poésie comme la création par le traducteur d'un poème nouveau dans sa propre langue: «What is important is what the source-language author has done with the sourcelanguage idiom and what the target-language writer will do with the target-language idiom. What matters, in other words, est le travail de l'écriture» (2007, p. 7). Il faut donc que le traducteur parcoure le même chemin que l'auteur de l'original pour donner forme à une pensée poétique, faire naître une ouvre nouvelle, et ce avec les ressources de sa propre langue.

Or, on voit que Bonnefoy, au fil de ses traductions de Shakespeare, ne se contente pas d'une recherche d'équivalence, 
mais s'efforce de faire texte, à son tour, en langue française et avec les ressources de sa poétique propre. Dans le cas de Hamlet, c'est par un acte de lecture sans cesse recommencé que Bonnefoy est parvenu à comprendre le texte, à le faire sien, et donc à composer une nouvelle œuvre de poésie. Selon Bonnefoy, le lecteur est celui «qui comprend le besoin d'échange de l'écrivain qu'il lit, assume ce besoin et dès lors assure ainsi à un être, cet écrivain, cette présence pleine que l'intuition poétique a rencontrée, plus brièvement, dans le monde» (2004, p. 72). Et si le lecteur peut participer de la recherche faite par le poète, il le fera «avec les moyens d'intensification et de vérification que lui donnerait sa propre faculté poétique s'il l'exerçait» (ibid.). Mais "pour ce faire il faut s'engager dans un travail d'écriture», nous dit Bonnefoy, laisser parler sa voix, rendre présente sa parole, ce que la simple lecture ne permet pas (ibid.). Ainsi, pour participer de la recherche poétique faite par Shakespeare dans Hamlet, Bonnefoy s'est-il fait traducteur, ce qui lui a permis de revivre cette même recherche à l'aune de sa propre sensibilité, et ce par un travail d'écriture véritable. Il a, à son tour, fait œuvre de poésie.

\section{Conclusion}

$\mathrm{Au}$ fil des lectures et des relectures de Hamlet, puis de ces réénonciations que sont les traductions, Bonnefoy est parvenu à toujours mieux entendre et comprendre la voix de Shakespeare pour, ultimement, lui répondre avec sa propre voix, celle du poète qu'il est. Comme l'explique Folkart, il a compris qu'il lui était impossible de réactualiser le «sens total» (comprenant sa signifiance, sa référence et ses contenus) d'un texte, conçu comme un absolu, mais que ce que tout traducteur réactualise "c'est, non pas un absolu, mais sa vision du texte, son modèle, le texte en interaction avec lui, une hypostase qui ne coïncide que partiellement avec l'énoncé sur lequel portent ses efforts» (1991, p. 333). À chaque étape du traduire, le traducteur interagit avec le texte de départ, que ce soit au moment de la lecture, qui est déjà interprétation, ou au moment de la re-médiation dans l'autre langue. En outre, le traducteur participe nécessairement à cette ré-énonciation qu'est la traduction, car il ne peut faire $f i$ de son expérience, de sa sensibilité, de sa subjectivité. Ainsi la voix de Bonnefoy interfère-t-elle nécessairement avec la voix de Shakespeare lorsqu'il traduit Hamlet. Les déviances qu'il fait subir 
au texte shakespearien et leur systématicité révèlent la présence créatrice de Bonnefoy au cœur des traductions ${ }^{19}$. Cependant, le fait que Bonnefoy ait produit plusieurs traductions de Hamlet, donc plusieurs ré-énonciations, qui révèlent des systématicités multiples ou croisées, illustre comment sa voix se modifie sans cesse. Tout se passe comme si Bonnefoy cherchait le timbre de sa voix au fil des traductions/ré-énonciations, ce qui suggère une certaine quête d'indépendance, un mouvement de la ré-énonciation vers la création. Au fil de ses relectures de Hamlet, Bonnefoy a identifié sa voix de traducteur, parallèlement à sa voix de poète pour écrire un texte nouveau en français, possédant sa propre organicité. Il est ainsi parvenu à faire acte de poésie.

\section{Références}

Berman, Antoine (1995). Pour une critique des traductions: John Donne. Paris, Gallimard, coll. «Bibliothèque des idées».

Berman, Antoine (1999). La Traduction et la Lettre ou l'Auberge du lointain. Paris, Seuil, coll. "L'Ordre philosophique».

Bernardet, Arnaud (2007). "La voix comme je ne sais quoi. "De la musique avant toute chose"?». Revue Verlaine, 10, p. 65-110.

Bonnefoy, Yves (1993a). «Traduire en vers ou en prose», Théâtre et poésie. Shakespeare et Yeats. Paris, Mercure de France, 1998, p. 209-220.

Bonnefoy, Yves (1993b). «Traduire la poésie (2). Entretien avec JeanPierre Attal (1993)», La Communauté des traducteurs. Strasbourg, Presses universitaires de Strasbourg, p. 59-67.

Bonnefoy, Yves (2000). «Avant-propos», La Communauté des traducteurs. Strasbourg, Presses universitaires de Strasbourg, p. 7-15.

Bonnefoy, Yves (2003). «Sur la traduction poétique» (entretien préparé par Pierre-Emmanuel Dauzat et Marc Ruggeri). In P.-E. Dauzat, dir. Cabier Steiner. Paris, Éditions de l'Herne, coll. «Cahiers de l'Herne", p. 201-215.

Bonnefoy, Yves (2004). «La traduction de la poésie ». Semicerchio, XXXXXXI, p. 62-80.

Dessons, Gérard (2004). L'art et la manière - art, littérature, langage. Paris, Honoré Champion.

Finck, Michèle (1989). Yves Bonnefoy, le simple et le sens. Paris, José Corti.

19. «Lorsque se fait jour une certaine systématicité de la déviation, lorsque la voix du traducteur conquiert une certaine autonomie, c'est que la traduction, sortant de ce degré zéro de la réappropriation qu'est le mimétisme, s'est faite confiscation, recréation ou même création». (Folkart, 1991, p. 398) 
Folkart, Barbara (1991). Le conflit des énonciations. Traduction et discours rapporté. Candiac, Éditions Balzac, coll. «L'univers des discours».

Folkart, Barbara (2007). Second Findings. A Poetics of Translation. Ottawa, University of Ottawa Press.

Meschonnic, Henri (1973). Pour la poétique II. Epistémologie de l'écriture. Poétique de la traduction. Paris, Gallimard, coll. «Le Chemin».

Meschonnic, Henri (1999). Poétique du traduire. Lagrasse, Verdier.

Roesler, Stéphanie (2010). Yves Bonnefoy et Hamlet. Thèse de doctorat, Département de langue et de littérature françaises, Université McGill. Inédit.

Shakespeare, William (1957). Cuvres complètes (Henry IV, Jules César, Comme il vous plaira, La Nuit des rois, Hamlet). Publiées sous la direction de Pierre Leyris et Henri Evans dans une traduction nouvelle accompagnée d'études, préfaces, notices, notes et glossaires. Paris, Formes et reflets.

Shakespeare, William (1959). Hamlet, Jules César. Trad. Yves Bonnefoy. Paris, Le Club français du livre.

Shakespeare, William (1962). Hamlet. Trad. Yves Bonnefoy. Paris, Mercure de France.

Shakespeare, William (1965). Hamlet. Trad. Yves Bonnefoy, version corrigée. Paris, Mercure de France.

Shakespeare, William (1978). Hamlet. Le Roi Lear. Préface et trad. Yves Bonnefoy, Paris, Gallimard, coll. «Folio ».

Shakespeare, William (1988). Hamlet. Trad. Yves Bonnefoy. Paris, Mercure de France.

Shakespeare, William (2004). Hamlet. Trad. J.M. Déprats. Préface, dossier et notes de G. Venet, établissement du texte anglais H. Suhamy. Gallimard, coll. «Folio Théâtre».

Thélot, Jérôme (1983). Poétique d'Yves Bonnefoy, Genève, Droz.

Venuti, Lawrence (1995). The Translator's Invisibility. Londres et New York, Routledge.

Vinay, Jean-Paul et Jean Darbelnet (1977 [1958]). Stylistique comparée du français et de l'anglais. Paris, Didier.

Stéphanie Roesler stephanie.roesler@mail.mcgill.ca 